\title{
UNIFORMLY CONVEX TOTALLY ORDERED SETS
}

\author{
R. H. REDFIELD ${ }^{1}$
}

ABSTRACT. Since a uniformly convex totally ordered set is a uniform lattice, its uniform completion is totally ordered in a natural way. The assumption of uniform convexity is a small restriction because every nearly uniform ordered space is uniformly convex.

1. Introduction. We investigated in [4] (which is summarized and discussed in [5]) how partially ordered sets equipped with certain types of Hausdorff uniformities may have their orders extended to their uniform completions. Since the outstanding example of this procedure is the extension of the order on the rational numbers to the real numbers, the question naturally arises whether the uniform completion of any totally ordered set with sufficiently restricted Hausdorff uniformity may be totally ordered.

In this note, we show that in the general setting of [4], total orders do indeed extend to total orders.

Terminology left undefined here may be found in [1], [2], and [6].

Let $X$ be a set. As usual, let $\Delta(X)=\{(x, x) \in X \times X \mid x \in X\}$ be the diagonal of $X$. Let $\mathfrak{U}$ be a uniformity on $X$. We denote the set of symmetric entourages of $\mathfrak{U}$ by $\mathfrak{U}^{s}$ and note that when partially ordered by inclusion, $\mathfrak{U}^{s}$ is a downwards directed set. Suppose that $\mathfrak{U}$ is Hausdorff, and let $(\mathbf{X}, \mathfrak{U})$ be the completion of $(X, \mathfrak{U})$ at $\mathfrak{U}$. Then instead of arbitrary Cauchy nets, we may restrict our attention to those with domain $\mathfrak{U}^{s}$ as follows: let $\mathbf{x} \in \mathbf{X}$ and let $\left\{y_{\delta} \mid \delta \epsilon\right.$ $\Delta\} \subseteq X$ be a Cauchy net converging to $x$. Then there exists a Cauchy net $\left\{x_{U} \mid U \in \mathfrak{U}^{s}\right\}$, with domain $\mathfrak{U}^{s}$, such that $\left\{x_{U}\right\}$ converges to $\mathbf{x}$ and such that, as subsets of $X,\left\{x_{U}\right\} \subseteq\left\{y_{\delta}\right\}$.

Let $(P, \leq)$ be a partially ordered set. We let

$$
G(\leq)=\{(x, y) \in P \times P \mid x \leq y\}
$$

be the graph of $\leq$. Let $\mathfrak{U}$ be a Hausdorff uniformity on $P$. Let $(P, \mathfrak{U})$ be the uniform completion of $(P, \mathfrak{U})$ at $\mathfrak{U}$. Define a binary relation on $\mathbf{P}$ as

Received by the editors August 9, 1973 and, in revised form, February 1, 1974.

AMS (MOS) subject classifications (1970). Primary 06A45, 54F05; Secondary 06A05, 54E 15 .

Key words and phrases. Uniform ordered space, totally ordered set, uniform completion, uniform lattice, uniformly convex.

1 The author would like to thank Norman Reilly for his encouragement and support during the development of these ideas. 
follows: $\mathbf{x} \leq \mathbf{y}$ if and only if there exist Cauchy nets $\left\{x_{U} \mid U \in \mathfrak{U}^{s}\right\} \subseteq P$ and $\left\{y_{U} \mid U \in \mathfrak{U}^{s}\right\} \subseteq P$ such that $\left\{x_{U}\right\}$ converges to $\mathbf{x},\left\{y_{U}\right\}$ converges to $\mathbf{y}$, and $x_{U} \leq y_{U}$ for all $U \in \mathfrak{U}^{s}$.

If $(L, \leq)$ is a lattice and if $\mathfrak{U}$ is a Hausdorff uniformity on $L$ with respect to which the lattice operations on $L$ are uniformly continuous, then we call $(L, \mathfrak{U}, \leq)$ a uniform lattice. The following was proved in [4].

Proposition 1.1. Let $(L, \mathbb{U}, \leq)$ be a uniform lattice with uniform completion $(\mathbf{L}, \mathfrak{U})$. Then $(\mathbf{L}, \mathfrak{U}, \preceq)$ is a uniform lattice sucb that the natural embedding $e$ of $(L, \mathfrak{U})$ into $(\mathbf{L}, \mathfrak{U})$ is a uniformly continuous lattice isomorphism. Furtbermore, $(\mathrm{L}, \mathfrak{U}, \preceq)$ satisfies the following universal mapping property: if $(\mathrm{K}, \mathfrak{B}, \leqq)$ is a uniformly complete uniform lattice and if $f:$ $(L, \mathfrak{U}, \leq) \rightarrow(K, \mathfrak{B}, \leqq)$ is a uniformly continuous order-preserving function, then there exists a unique uniformly continuous order-preserving function $\mathrm{f}$ : $(\mathbf{L}, \mathfrak{u}, \preceq) \rightarrow(\mathbf{K}, \mathfrak{B}, \leqq$ sucb that ef $=f$.

(We note that uniform lattices are a particularly well-behaved case. The analogous result for partially ordered sets requires a subtler definition of the extended partial order (see [4] or [5]).)

2. Uniform convexity. Let $(X, \mathfrak{U})$ be a uniform space. We let $T(\mathfrak{U})$ denote the topology on $X$ associated with $\mathfrak{U}$.

In a partially ordered set $(P, \leq)$, a subset $A \subseteq P$ is said to be convex if $a \leq x \leq b$ and $a, b \in A$ imply $x \in A$. Let $\mathfrak{U}$ be a uniformity on $P$. We say that $(P, \mathfrak{U}, \leq)$ (or just $\mathfrak{U}$ ) is locally convex if $x \in U \in T(\mathfrak{U})$ implies that there exist $V \in T(\mathfrak{l})$ and a convex subset $C \subseteq P$ such that $x \in V \subseteq C \subseteq U$, i.e. if every neighbourhood contains a convex neighbourhood. We say that $(P, \mathfrak{U}, \leq$ ) (or just $\mathfrak{U}$ ) is uniformly convex if for all $U \in \mathfrak{U}$ there exists $V \in$ $\mathfrak{U}$ such that $V \subseteq U$ and $V[x]$ is convex for all $x \in P$. Clearly if $(P, \mathfrak{U}, \leq)$ is uniformly convex, then it is locally convex.

Example 2.1. In this example we construct a locally convex, nonuniformly convex, Hausdorff uniformity on a totally ordered set.

Let $R$ be the real numbers, let $Q$ be the rational numbers, and let $N$ be the natural numbers. Partially order $R \times R$ by: $(x, y) \leq(a, b)$ if and only if $x \leq a$ and $y \leq b$. For $n \in N$, define $U_{n}, V_{n}, W_{n} \subseteq R \times R$ by

$$
\begin{aligned}
& U_{n}=\{(x, y) \mid(x, y) \leq(0,0) \text { and }|y-x| \leq 1 / n\} \cup \Delta(R), \\
& V_{n}=\{(x, y) \mid(x, y) \geq(n, n) \text { and }(x, y) \in 2 \times Q\} \\
& W_{n}=U_{n} \cup V_{n^{*}}
\end{aligned}
$$


Clearly $\Delta(R) \subseteq W_{n}$ and $W_{n}^{-1}=W_{n}$ for all $n$. Let $(x, y),(y, z) \in W_{2 n}$. If $x=y$ or $y=z$, then $(x, z) \in W_{2 n} \subseteq W_{n^{*}}$ Otherwise, $x \neq y$ and $y \neq z$. If $(x, y) \in U_{2 n}$, then $(x, y) \leq(0,0)$ and $|y-x| \leq 1 / 2 n$. Since then $y \leq 0$, $(y, z) \notin V_{2 n}$. Thus $(y, z) \in U_{2 n},(y, z) \leq(0,0)$, and $|z-y| \leq 1 / 2 n$. Then $(x, z) \leq(0,0)$ and

$$
|z-x| \leq|z-y|+|y-x| \leq 1 / 2 n+1 / 2 n=1 / n
$$

Thus $(x, z) \in U_{n} \subseteq W_{n}$. If $(x, y) \in V_{2 n}$, then $(x, y) \geq(2 n, 2 n)>(0,0)$ and hence, as above, $(y, z) \in V_{2 n}$. Since $x \neq y \neq z, x, y, z \in Q$ and thus $(x, z)$ $\epsilon Q \times Q$. Hence $(x, z) \in V_{2 n} \subseteq W_{n^{*}}$. Therefore, $W_{2 n} \circ W_{2 n} \subseteq W_{n^{*}}$ Thus, $\left\{W_{n} \mid n \in N\right\}$ is a filter-base for a uniformity $\mathfrak{U}$ on $R$. Clearly $\mathfrak{U}$ is locally convex and Hausdorff, but not uniformly convex. We note also that $T(\mathbb{I I})$ is not discrete.

We will prove our main result for uniformly convex uniformities. This is no great restriction, however, since we will also show that the general setting which allows the order to be extended in fact entails uniformly convex uniformities.

Proposition 2.2. Let $(T, \leq)$ be a totally ordered set. If $\mathfrak{U}$ is a uniformly convex Hausdorff uniformity on $T$, then $(T, \mathfrak{U}, \leq)$ is a uniform lattice.

Proof. Let $U \in \mathfrak{U}$. Let $W \in \mathfrak{u}^{s}$ be such that $W \circ W \subseteq U$. Let $V \in \mathfrak{U}$ be such that $V[x]$ is convex for all $x \in T$ and $V \subseteq W$. Let

$$
V^{*}=\{(a, x, b, y) \mid(a, b),(x, y) \in V\} \text {. }
$$

It suffices to show that $V^{*}(\vee \times \bigvee) \subseteq U, V^{*}(\Lambda \times \wedge) \subseteq U$. We will prove the first containment; the second may be proved similarly. Let $(a, x, b, y) \epsilon$ $V^{*}$. If $a \geq x$ and $b \geq y$ or if $a \leq x$ and $b \leq y$, then $(a \vee x, b \vee y) \in V \subseteq U$. Otherwise, $x \geq a$ and $y \leq b$, or $x \leq a$ and $y \geq b$. Suppose $x \leq a$ and $y \geq b$. Then $(a, x, b, y)(\vee \times \bigvee)=(a, y)$.

(1) Suppose $y \geq a$ : Then $y \geq a \geq x$ implies $a \in V[x]$ since $y \in V[x]$ and $V[x]$ is convex. Thus $(x, a) \in V$, i.e. $(a, x) \in V^{-1}$. Hence

$$
(a, y) \in V^{-1} \circ V \subseteq W^{-1} \circ W=W \circ W \subseteq U \text {. }
$$

(2) Suppose $y<a$ : Then $a>y \geq b$ implies $y \in V[a]$ since $b \in V[a]$ and $V[a]$ is convex. That is, $(a, y) \in V \subseteq U$. Similarly one may show that if $x \geq a$ and $y \leq b$, then $(a, x, b, y)(\bigvee \times \bigvee)=(x, b) \in U$. Therefore, $V^{*}(\mathrm{~V} \times \mathrm{V}) \subseteq U$

Theorem 2.3. Let $(T, \leq)$ be a totally ordered set. Let $\mathfrak{U l}$ be a uniformly convex Hausdorff uniformity on $T$. Then $(\mathrm{T}, \preceq)$ is a totally ordered set. 
Proof. By Proposition 2.2, $(T, \mathfrak{U}, \leq)$ is a uniform lattice, and hence by Proposition 1.1 , we may treat $(T, \leq)$ as a sublattice of the uniform lattice $(\mathbf{T}, \mathfrak{u}, \preceq)$.

Let $\mathbf{x}, \mathbf{y} \in \mathbf{T}$. Let $\left\{x_{U} \mid U \in \mathfrak{U}^{s}\right\} \subseteq T$ be a Cauchy net converging to $\mathbf{x}$ and let $\left\{y_{U} \mid U \in \mathfrak{U}^{s}\right\}$ be a Cauchy net converging to $y$. Let $\Delta=\left\{U \in \mathfrak{U}^{s}\right\}$ $\left.x_{U} \geq y_{U}\right\}$. Suppose first that $\Delta$ is cofinal in $\mathfrak{U}^{s}$. Then $\left\{x_{U} \mid U \in \Delta\right\}$ converges to $\mathbf{x}$ since $\mathfrak{i l}$ is Hausdorff. Thus, since $x_{U} \vee y_{U}=x_{U}$ for all $U \in \Delta,\left\{x_{U} \vee y_{U} \mid U \in \Delta\right\}$ converges to $\mathbf{x}$. However, since $(\mathbf{T}, \mathfrak{u}, \preceq)$ is a uniform lattice, $\left\{x_{U} \vee y_{U} \mid U \in \mathfrak{U}^{s}\right\}$ converges to $\mathbf{x} \vee \mathbf{y}$, and thus, since $\Delta$ is cofinal, $\left\{x_{U} \vee y_{U} \mid U \in \Delta\right\}$ converges to $\mathbf{x} \vee \mathbf{y}$. Since $\mathfrak{U}$ is Hausdorff, we conclude that $x \vee y=x$, i.e., that $y \preceq x$. Now suppose that $\Delta$ is not cofinal in $\mathfrak{U}^{s}$. Then there exists $V \in \mathfrak{U}^{s}$ such that for all $U \subseteq V, x_{U} \geq y_{U}$, i.e., since $(T, \leq)$ is totally ordered, $x_{U}<y_{U}$. Let

$$
z_{U}= \begin{cases}x_{U} & \text { if } U \subseteq \underline{\not} V, \\ y_{U} & \text { if } U \subseteq V .\end{cases}
$$

Clearly $x_{U} \leq z_{U}$ for all $U \in \mathfrak{U}^{s}$ and $\left\{z_{U} \mid U \in \mathfrak{M}^{s}\right\} \subseteq T$ is a Cauchy net converging to $y$. Thus $x \preceq y$, and therefore $(\mathbf{T}, \preceq)$ is totally ordered.

Corollary 2.4. Let $(T, \leq)$ be a totally ordered set, and id a uniformly convex Hausdorff uniformity on $T$. Let $(\mathrm{S}, \leqq)$ also be a totally ordered set and suppose that $B$ is a uniformly convex Hausdorff uniformity on $S$ such that $(\mathrm{S}, \mathfrak{Z})$ is uniformly complete. If $f:(T, \mathfrak{u}, \leq) \rightarrow(\mathrm{S}, \mathfrak{Z}, \leqq)$ is a uniformly continuous order-preserving function, then there exists a unique uniformly continuous order-preserving function $\mathrm{f}:(\mathrm{T}, \mathfrak{u}, \leqq) \rightarrow(\mathrm{S}, \mathfrak{B}, \leqq)$ such that ef $=f$, where $e$ is the canonical embedding of $(T, \mathfrak{U})$ into $(\mathbf{T}, \mathfrak{U})$.

Proof. The result follows from Proposition 1.1 and Theorem 2.3.

3. Uniform ordered spaces. In [4] we showed that a natural setting for extending a partial order on a uniform space to its uniform completion is the category of uniform ordered spaces and uniformly continuous orderpreserving functions. Nachbin [3] proved that every uniform ordered space is locally convex. Using a similar argument, we will show that every nearly uniform ordered space is in fact uniformly convex. Thus, by Theorem 2.3, the natural order on the uniform completion of a totally ordered uniform ordered space will be a total order.

Let $X$ be a set. A semiuniform structure [3] for $X$ is a filter $\mathcal{F}$ on $X \times X$ satisfying 
(i) $\Delta(X) \subseteq V$ for all $V \in \mathcal{F}$;

(ii) for all $V \in \mathcal{F}$, there exists $U \in \mathcal{F}$ such that $U \circ U \subseteq V$. Thus, a semiuniform structure is almost a uniformity. We can add the condition of symmetry by considering

$$
\mathfrak{F}^{*}=\left\{U \cap V^{-1} \mid U, V \in \mathcal{F}\right\},
$$

which is easily seen to be a uniformity on $X$ [3].

A nearly uniform ordered space [4] is a partially ordered set $(P, \leq)$ with a Hausdorff uniformity $\mathfrak{l}$ on $P$ such that there exists a semiuniform structure $\mathfrak{F}$ for $P$ with $\cap \mathcal{F} \supseteq G(\leq)$ and $\mathfrak{F}^{*}=\mathfrak{U}$. A uniform ordered space [3] is a partially ordered set $(P, \leq)$ with a Hausdorff uniformity $\mathfrak{U}$ and semiuniform structure $\mathfrak{F}$ such that $\cap \mathcal{F}=G(\leq)$ and $\mathfrak{F}^{*}=\mathfrak{U}$.

Proposition 3.1. Every nearly uniform ordered space is uniformly convex.

Proof. Let $(P, \leq)$ be a partially ordered set; let $\mathfrak{U}$ be a Hausdorff uniformity on $P$; and suppose that $\mathcal{F}$ is a semiuniform structure for $P$ such that $\cap \mathcal{F} \supseteq G(\leq)$ and $\mathcal{F}^{*}=\mathfrak{U}$. Let $U \in \mathfrak{U}$. Since $\mathfrak{F}^{*}=\mathfrak{U}$, there exists $F \in \mathfrak{F}$ such that $F \cap F^{-1} \subseteq U$. Let $V \in \mathcal{F}$ be such that $V \circ V \subseteq F$, and let $W=$ $V \cap V^{-1}$. If $A \subseteq P$, let

$$
\operatorname{Conv}(A)=\{t \in P \mid \text { there exist } a, b \in A \text { with } a \leq t \leq b\} .
$$

Clearly, for all $A \subseteq P$, Conv $(A)$ is a convex set containing $A$. For all $x \in P$, let $L_{x}=\operatorname{Conv}(W[x])$. Finally, let $M=\left\{(x, y) \in P \times P \mid y \in L_{x}\right\}$. Clearly, for all $x \in P, M[x]=L_{x}$ is convex. Thus it suffices to show that $M \in \mathfrak{l}$ and that $M \subseteq U$. If $(x, y) \in W$, then $y \in W[x] \subseteq L_{x}$ and hence $(x, y) \in M$. Thus $W \subseteq M$, and hence $M \in \mathfrak{U}$.

Let $(x, y) \in M$. Then $y \in L_{x}$, and hence there exist $a, b \in W[x]$ such that $a \leq y \leq b$. Now $(x, a) \in W \subseteq V,(a, y) \in G(\leq) \subseteq V$. Thus $(x, y) \in V \circ V$ $\subseteq F$. Also, $(x, b) \in W \subseteq V^{-1},(y, b) \in G(\leq) \subseteq V$. Thus $(b, x) \in V$, and hence $(y, x) \in V \circ V \subseteq F$. Therefore, $(x, y) \in F \cap F^{-1} \subseteq U$, and hence $M \subseteq U$.

Corollary 3.2. Let $(P, \mathfrak{U}, \leq)$ be a uniform ordered space. If $(P, \leq)$ is totally ordered, then $(\mathbf{P}, \preceq)$ is totally ordered.

Proof. The result follows from Theorem 2.3 and Proposition 3.1.

We conclude this final section by noting that, in the totally ordered case, most of the different types of spaces that have been mentioned are equivalent. Thus, the converse of Proposition 2.2 holds, as does that of Proposition 3.1 for totally ordered sets. 
Proposition 3.3. Let $(T, \leq)$ be a totally ordered set; let $\mathfrak{U}$ be a uniformity on $T$. Then the following statements are equivalent:

(i) $(T, \mathfrak{U}, \leq)$ is a uniform ordered space;

(ii) $(T, \mathfrak{U}, \leq)$ is a nearly uniform ordered space;

(iii) $\mathfrak{U}$ is Hausdorff and uniformly convex;

(iv) $(T, \hat{i}, \leq)$ is a uniform lattice.

Proof. That (i) implies (ii) is clear (cf. [4, Proposition 2.4]). By Proposition 3.1, (ii) implies (iii). By Proposition 2.2, (iii) implies (iv), and finally by [3, Proposition 11], (iv) implies (i).

\section{REFERENCES}

1. G. Birkhoff, Lattice theory, 3rd ed., Amer. Math. Soc. Colloq. Publ., vol. 25, Amer. Math. Soc., Providence, R. I., 1967. MR 37 \# 2638.

2. N. Bourbaki, Eléments de mathématique. I: Les structures fondamentales de l'analyse. Livre III: Topologie générale, Actualités Sci. Indust., nos. 1029, 1045, 1084, 1142, 1143, Hermann, Paris, 1947, 1948, 1949; English transl., Hermann, Paris; Addison-Wesley, Reading, Mass., 1966. MR 34 \# 5044b.

3. L. Nachbin, Topology and order, Van Nostrand Math. Studies, no. 4, Van Nostrand, Princeton, N. J., 1965. MR 36 \#2125.

4. R. H. Redfield, Ordering uniform completions of partially ordered sets, Canad. J. Math. 26 (1974), 644-664.

5. - Ordering uniform completions of partially ordered sets, Lattice Theory, Proc. University of Houston Conf., Math. Dept., Univ. of Houston, Houston, Tex., 1974, pp. 504-511.

6. W. Thron, Topological structures, Holt, Rinehart and Winston, New York, 1966. MR $34 \# 778$.

DEPARTMENT OF MATHEMATICS, SIMON FRASER UNIVERSITY, BURNABY 2, BRITISH COLUMBIA, CANADA

Current address: Department of Mathematics, Monash University, Clayton, Victoria, Australia 